A N N A L E S Annales de Bretagne et des Pays de l'Ouest

Anjou. Maine. Poitou-Charente. Touraine

$121-1 \mid 2014$

Varia

\title{
Dictionnaire des corsaires et pirates
}

\section{Olivier Chaline}

\section{(2) OpenEdition}

Journals

Édition électronique

URL : http://journals.openedition.org/abpo/2749

DOI : $10.4000 / a b p o .2749$

ISBN : 978-2-7535-3407-0

ISSN : 2108-6443

Éditeur

Presses universitaires de Rennes

Édition imprimée

Date de publication : 27 mars 2014

Pagination : 196-197

ISBN : 978-2-7535-3405-6

ISSN : 0399-0826

Référence électronique

Olivier Chaline, "Dictionnaire des corsaires et pirates », Annales de Bretagne et des Pays de l'Ouest [En ligne], 121-1 | 2014, mis en ligne le 27 mars 2014, consulté le 23 septembre 2020. URL : http:// journals.openedition.org/abpo/2749; DOI : https://doi.org/10.4000/abpo.2749 
Avec le veuvage, la femme retrouve sa pleine capacité juridique. Anaïs Dufour note dans le chapitre 4 l'écrasante majorité des veuves seigneurs : elles représentent 90 \% des rôles de l'arrière-ban et 74 \% des aveux (p. 78). En cas d'enfants mineurs, les veuves peuvent être gardiennes ou tutrices. Toutefois, elles restent vulnérables et soumises à la surveillance familiale. La viduité est souvent un temps de précarité et nombre de veuves préfèrent s'en remettre à des proches ou des procureurs royaux pour administrer la seigneurie. D'autres au contraire se comportent comme des seigneurs, vigilantes quant à leurs droits seigneuriaux. Dans ce portrait des dames, se distinguent les " haultes et puissantes princesses " analysées au chapitre 5. Certaines titulaires d'un duché-pairie le reçoivent parfois en récompense des services rendus à la monarchie; la plupart en héritent lorsqu'il est tombé en quenouilles faute d'héritier mâle car la dérogeance au principe de masculinité est tolérée jusqu'en 1711. La dévolution de ces fiefs est étroitement surveillée par le pouvoir royal comme le montre l'exemple de la transmission du comté d'Eu après la mort de Jacques de Clèves, comte de Nevers en 1564. Ensuite, Anaïs Dufour examine la manière dont ces grandes dames gèrent leur seigneurie en présentant l'exemple de la duchesse de Longueville attentive à ses prérogatives de seigneur haut justicier puis celui de la baronne de Ferrières épouse séparée de biens qui se révèle une redoutable gestionnaire. Enfin, l'auteur analyse leur rôle politique dans le contexte troublé de la Ligue à partir du cas de Marie de Lorraine duchesse d'Aumale. L'ouvrage s'achève sur 31 pages de pièces justificatives composées d'aveux, de lettres de dons, d'articles de la coutume et de signatures. Une liste des sources, une bibliographie et un index des noms de lieux et de personnes complètent utilement l'ensemble.

Au final, l'auteur livre un travail riche et bien écrit. Elle apporte un regard intéressant sur la seigneurie au féminin et une étude minutieuse de la coutume normande.

Aurélie ChATENET-CALYSTE

ButI, Gilbert, HRODEJ, Philippe (dir.), Dictionnaire des corsaires et pirates, Paris, CNRS éditions, 2013, 990 p.

À la tête d'un équipage qui n'est pas de forbans (et qu'on découvre au fil des notices avant que son rôle soit donné in fine), Gilbert Buti et Philippe Hrodej nous livrent, sous la forme d'un dictionnaire comptant presque 600 entrées, le fruit d'une patiente enquête portant sur une vaste époque moderne, du $\mathrm{Xvl}^{\mathrm{e}}$ au début du XIXe siècle. Même si l'écho de l'actualité marquée par le réveil de la piraterie çà et là sur les mers du globe retentit dans ce volume, il a été sage de ne pas vouloir y inclure les temps contemporains qui apparaissent toutefois avec des entrées sur la Somalie des années 1990-2012, le détroit de Malacca ou encore le golfe de Guinée. Le propos est déjà fort ambitieux puisqu'il s'agit, nous disent les deux directeurs, de " saisir le rôle historique de la course, de la piraterie et de la flibuste en pointant les espaces qui ont été les plus florissants, les terrains de chasse les mieux pourvus et les périodes les plus fertiles, en restituant des parcours individuels et collectifs, connus ou non, en réveillant des "dynasties", comme celle des Doria, et en suivant des 'météores aux réussites fulgurantes' à l'image de Noël Danycan ".

La diversité des entrées rend bien compte de ce programme. Aux notices biographiques très nombreuses et attendues sur des célébrités aux parcours exceptionnels ou des inconnus que leur absence de notoriété n'empêche pas d'être représentatifs, s'en ajoutent d'autres portant sur des ports, des îles ou zones géographiques, voire sur des objets (pavillons pirates et corsaires). Ce dictionnaire a aussi l'avantage de 
fournir un glossaire bien venu, un catalogue des sources (imprimées et manuscrites) ainsi qu'une bibliographie substantielle et classée par périodes puis siècles pour les temps modernes. Autant dire que l'on dispose d'un véritable instrument de travail paré pour être utilisé aussi bien par des chercheurs que par les passionnés d'histoires de corsaires ou de pirates.

Les cartes données dès le début du volume, font bien saisir que la scène est le monde, toutes les mers et tous les océans sont pris en compte, avec des articles synthétiques apportant une information parfois difficile à trouver, ainsi sur Londres ou sur la mer de Sulu. On regrettera simplement qu'il n'y ait pas un système de renvoi, par exemple par des numéros, vers les ouvrages cités dans la bibliographie. Le souci de ne rien manquer d'essentiel a parfois conduit les maîtres d'œuvre à se charger eux-mêmes de notices pour lesquelles ils avaient pu peiner à trouver un auteur, ainsi Philippe Hrodej nous donne-t-il un article sur le port adriatique de Senj (Segna), un des points de départ des Uscoques contre le commerce vénitien ainsi qu'un autre sur la sitch des cosaques zaporogues qui, depuis le Dniepr, opéraient en Mer Noire au XVII ${ }^{\mathrm{e}}$ siècle.

Le parti a été pris de traiter le phénomène de l'insécurité maritime à l'époque moderne dans sa globalité, quels qu'en aient été les modalités et les acteurs. Course, flibuste et piraterie se trouvent donc envisagées comme autant de facettes d'une même réalité. Aussi ne faut-il pas être surpris outre mesure de trouver des voisinages peut-être quelque peu inattendus entre des capitaines corsaires issus de respectables familles malouines et des gens objectivement de sac et de corde qui finissent parfois pendus, entre le cardinal de Richelieu et des renégats installés en Afrique du Nord, entre des officiers de la marine royale et des négriers... La lutte contre la piraterie n'est pas absente de ce volume, comme en témoigne, par exemple, la notice consacrée à Stephen Decatur, le héros de l'US Navy contre les Barbaresques au début du $\mathrm{XIX}^{\mathrm{e}}$ siècle. Les gagnants, au premier rang desquels quelques illustres lignées malouines, voisinent avec des perdants, victimes du bras de Thémis ou laissés invalides, tel ce Mathieu Delarue, granvillais qui survit, aveugle et muet, le visage broyé dissimulé par un masque de métal...

Au-delà des fortunes de mer et des campagnes réussies, des violences infligées ou subies, beaucoup d'entrées, notamment celles sur des ports mais aussi certaines davantage biographiques, nous conduisent vers la place de ces activités prédatrices dans la vie des économies et des sociétés comme vers les liens qu'elles entretiennent avec les États et leurs instruments militaires. Placements (de reconversion ou non), occasions de promotion sociale ou de rétablissement d'une position compromise (René-Auguste de Chateaubriand a sa notice), choix stratégiques articulant escadres et guerre au commerce sont autant d'aspects, aujourd'hui mieux connus grâce à des décennies de recherches, sur lesquels ce substantiel dictionnaire ouvre des perspectives. Par les quelques remarques formulées dans l'introduction, il appelle une réflexion renouvelée sur le rapport entre la violence (ici sous la forme de la prédation, avec ou sans guerre) et les affaires. Dans cette direction, le récent ouvrage de David Parrott, The Business of War. Military Entreprise and Military Revolution in Early Modern Europe, Cambridge, Cambridge University Press, 2012, qui a toute une dimension maritime avec des personnages et des groupes que l'on retrouve dans le Dictionnaire, pourrait permettre d'aller plus avant.

Olivier CHALINE 